\title{
Moving Toward the Light: Using New Technology to Answer Old Questions
}

\author{
JENNIFER L. LUCITTI AND MARY E. DICKINSON
}

Department of Molecular Physiology and Biophysics, Baylor College of Medicine, Houston, Texas, 77071

\begin{abstract}
Fluorescence microscopy has become a principle methodology in the field of developmental biology. Recent technological advances have led to the design of high-speed and highresolution confocal and multiphoton microscopes that enable researchers to obtain three- and four-dimensional information in living cells and whole embryos. Paralleling this progress, the development of stable and bright vital fluorescent probes has revolutionized the ability to track individual cells in vitro and in vivo and to visualize intercellular and subcellular molecular interactions in real time. Combining imaging modalities and labeling techniques that are increasingly unobtrusive to cell and whole animal function, our understanding of how proteins interact, tissues take form, and organs synchronize to create a functioning animal is reaching a whole new level. (Pediatr Res 60: 1-5, 2006)
\end{abstract}

$\mathrm{M}^{\mathrm{i}}$ icroscopy has been an essential tool in the investigation of developmental processes for three centuries. As technology progresses, old questions can be answered in new ways and new questions emerge. Two areas in particular have shown tremendous progress: microscopes and imaging technology, and the development of more advanced fluorescent probes and markers. Advances in high-resolution computeraided microscopes, including confocal and multiphoton microscopes, enable researchers to collect detailed threedimensional reconstructions of changes in morphology from thick tissues and embryos. Adding to the tools available to microscopists, there has been an explosion of bright, stable, vital fluorescent probes for studying molecular processes in living cultured cells, embryos, and whole animals. These advances include the development of a virtual rainbow of genetically encoded fluorescent proteins, as well as the emergence of quantum dots, molecular beacons, and next-generation small molecule dyes. This review discusses some of the emerging technology used to study the mechanisms of embryogenesis.

\section{MICROSCOPES AND IMAGING TECHNOLOGY}

Fluorescence imaging in living embryonic models can be challenging. Incident and fluorescent light is often absorbed

Received December 13, 2005; accepted December 14, 2005.

Correspondence: Mary E. Dickinson, Ph.D., Baylor College of Medicine, Department of Molecular Physiology and Biophysics, One Baylor Plaza, Houston, TX 77071; e-mail: mdickins@bcm.edu

DOI: 10.1203/01.pdr.0000220318.49973.32 and scattered by the sample, decreasing the ability to resolve structural and molecular detail deep in the sample, and autofluorescence can often challenge our ability to identify fluorescent structures. Several methods have emerged to reduce artifacts from scattered light, such as deconvolution methods, but perhaps none are more popular than the use of LSM. Confocal, multiphoton, and multispectral LSM have become available on many campuses and in many labs, providing advanced imaging tools to many cell and developmental biologists.

The principle of confocal microscopy is relatively simple. Lasers used as excitation sources are scanned as a focused point across the sample. Fluorescence emission resulting from the excitation light is collected back through the objective lens and passes through a pinhole before reaching the detector. The pinhole allows light from the focal plane to reach the detector but rejects the out-of-focus photons from contributing to noise in the image. By scanning a sample in fixed increments in the z-plane, positional information of molecular markers in cells and tissues can be reconstructed in three dimensions. In combination with computational advances in three-dimensional reconstruction and image segmentation, confocal microscopy has become a powerful tool for molecular imaging and can be combined with time-lapse microscopy to provide a four-dimensional view of developing cells and tissues.

Multiphoton microscopy offers several advantages over confocal microscopy when imaging living tissue. With this technology, fluorophore excitation occurs after the nearsimultaneous absorption of two or more photons. Photons of relatively long wavelength, which singly may not excite a specific fluorophore, can be "combined" to produce enough energy to achieve dye excitation. Using this method, excitation of the dye is only achieved at the focal plane, where the photon flux density is high enough for the near-simultaneous absorption of two or more photons. Ultrafast lasers emitting high-intensity, near-infrared pulses are used to deliver instantaneous bursts of light to the tissue increasing the probability

Abbreviations: EGF, epidermal growth factor; FP, fluorescent protein; FRET, Forster or fluorescence resonance energy transfer; GFP, green fluorescent protein; LSM, laser scanning microscopy; MB, molecular beacons; QD, quantum dot 
of a two-photon absorption event. Because fluorochromes are not excited outside the focal plane, a pinhole is not needed to acquire optical sections; inherent optical sectioning capability is provided by the nonlinear effect. The advantages of multiphoton microscopy are numerous. The longer excitation wavelength used and the reduced absorption and scattering of incident light enables efficient imaging at deeper levels within the tissue than with confocal microscopy. For instance, images taken at depths two or more times greater than that accessible with confocal microscopy have been demonstrated by Centonze and White (1). Another advantage of this method is that near-infrared light is less harmful to tissues and the lack of out of focus excitation reduces the overall exposure of the tissue to phototoxic effects produced by fluorochrome bleaching. Together, these properties make multiphoton microscopy an excellent tool for live, three-dimensional or four-dimensional imaging, but this approach does have the drawback that the combined price tag of the microscope system and the ultrafast laser can be prohibitive.

Both confocal and multiphoton microscopy were initially developed as point scanning systems, where data are collected pixel-by-pixel across an entire line, then pixel-by-pixel across the next line, etc. Although this is an efficient and reliable way to excite and collect photons, it is fairly slow: an image every few hundred milliseconds or every few seconds depending on the signal-to-noise that is required. For fast motions, such as ion release, heart contractions, or blood flow, the sampling speed is too slow for accurate data collection. To overcome these problems, multispot detection or slit-scanning confocals have emerged that rely on illuminating and collecting emission from multiple spots at once. Some examples of commercially available multipoint scanners are the PerkinElmer (Norwalk, CT) Ultraview spinning disk confocal microscope, or the Zeiss (Carl Zeiss Inc., Thornwood, NY) LSM 5 LIVE confocal microscope, or for multiphoton excitation, LaVision's TriMScope. The image acquisition speed on these systems can range from video rate (24 frames/s) up to $\mathrm{KHz}$ frame rates in some instances.

Another exciting advance in laser scanning microscopy has been the emergence of microscopes with sophisticated spectrometers and analysis packages for the simultaneous imaging of multiple fluorochromes and the elimination of autofluorescence. Whereas confocal microscopes, like wide-field microscopes, have relied on changeable glass filters for spectral separation of multiple fluorochromes, LSM equipped with multispectral detectors, such as the META detector (Zeiss) or the SP system (Leica, Wetzlar, Germany) are capable of acquiring the full spectrum of emission signal from a sample. The contribution of individual fluorochromes can then be determined using linear unmixing algorithms that determine the relative contribution of each fluorochrome on a pixel-bypixel basis by matching the spectral output to a series of known ratios of linearly mixed dye combinations. This method not only enables more dyes to be used in a single experiment, but can also provide a means to identify and eliminate autofluorescence that does not match the spectra of the dyes used in the experiment (2).

\section{ENLIGHTENMENT FROM FP-LABELED EMBRYOS}

The discovery of FP has no doubt had a tremendous impact on the vital imaging and developmental biology. These stable, colorful, and versatile markers have made it possible to study dynamic interactions between and within cells in living organisms. Since the isolation of the first FP, GFP, a long list of mutated, multi-color counterparts have been described, allowing researchers a virtual rainbow of possibilities to use as cellular reporters. It has become commonplace to engineer organisms from bacteria to mice with glowing jellyfish proteins. Placed under the control of a cell-type specific promoter, FP can act as a convenient and bright cellular marker. Fused to other proteins, FP can be used to label specific subcellular compartments such as the nucleus, mitochondria, or cell membrane or to follow the intracellular dynamics of a protein of interest. For instance, histone-2B/FP fusions have proven very useful for studying cell division as the fluorescent fusion brightly marks chromosomal dynamics during division (3). It has also been used to track cells in vivo because the bright, concentrated fluorescence in the nucleus makes it possible to identify and follow single cells (3) (Fig. 1). The growing availability of cell-type specific promoters in many systems and the ease of tagging individual proteins of interest makes this labeling technology one of the most widely used.

Perhaps the greatest impact from imaging studies using FP has been in the fields of cardiovascular and neural development. In both zebrafish and mouse, fluorescently labeled endothelial cell lines have been used to study vasculogenesis and angiogenesis. In zebrafish, GFP expression controlled by the endothelial-specific flil promoter has been imaged using

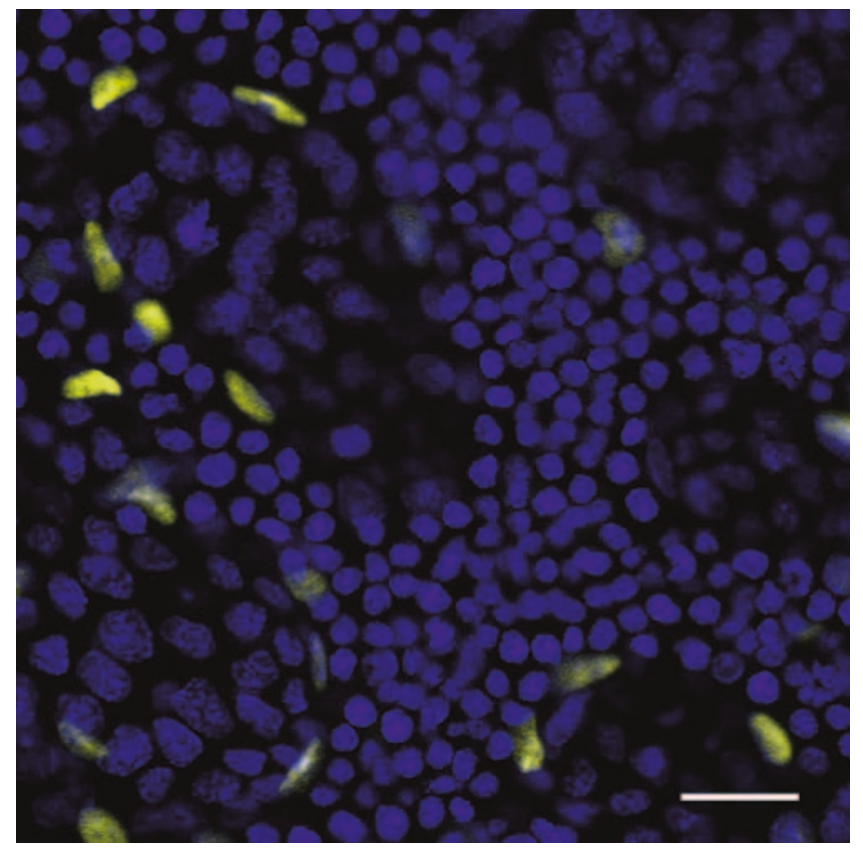

Figure 1. Whole-mount image of an $8.5 \mathrm{dpc}$ murine embryo yolk sac expressing H2B:YFP under the control of the Flk-1 promoter. Endothelial cells, easily identified by their yellow fluorescent nuclei, line the honeycomblike capillary network. Erythroblasts, identified by their small, round nuclei, densely populate the capillary network and release into circulation during vascular remodeling. Bar is $20 \mu \mathrm{m}$. (Image courtesy of Monica Garcia.) 
confocal or multiphoton microscopy to study patterns of angiogenesis in three spatial dimensions over time (so called four-dimensional imaging) in normal $(4,5)$ and mutant strains, showing that the establishment of interconnections between vessels and the specification of arterial/venous identity required hemodynamic cues (6). GFP expressed under the flil promoter also leads to expression in the endocardium and endocardial cushions, making it possible to study valve development in normal and mutant fish, such as those carrying the silent heart mutation. Recent studies using fast-acquisition confocals have enabled real-time imaging of valve primordia and GFP labeled blood cells have been imaged using this high-speed technology to determine when valve cushions are capable of preventing the regurgitative flow between chambers that is seen once the heart starts looping (7).

Similar to the present capabilities in zebrafish, the recent development of methods to culture and image mouse embryos with laser scanning microscopy has enabled the direct observation of early dynamic events in mammalian development (Fig. 2). Vital markers of angiogenesis have been created by placing GFP under the control of the Tie-2 promoter or H2B-YFP under the control of the Flk-1 promoter and using GFP-labeled erythroblasts, circulation of the very first blood cells from the yolk sac to the embryo has been directly visualized (3). Such markers make it possible to not only observe the movement of blood cells but to measure how fast they move (Fig. 3). It has long been thought that angiogenesis depends on the circulation of blood through developing vessels by imparting force on the vessel walls. Using fast-line scanning on a traditional confocal microscope or rapid-frame scanning on the new LSM 5 LIVE system, the velocities of circulating GFP-labeled blood cells have been measured and estimates of the wall shear stress were calculated, showing that shear stress levels encountered during vessel remodeling are similar to those known to cause changes in cell alignment and the up-regulation of growth factors needed for vessel stabilization and pericyte recruitment (8). Thus, rapid confocal imaging technology has made it possible to calculate hemo-
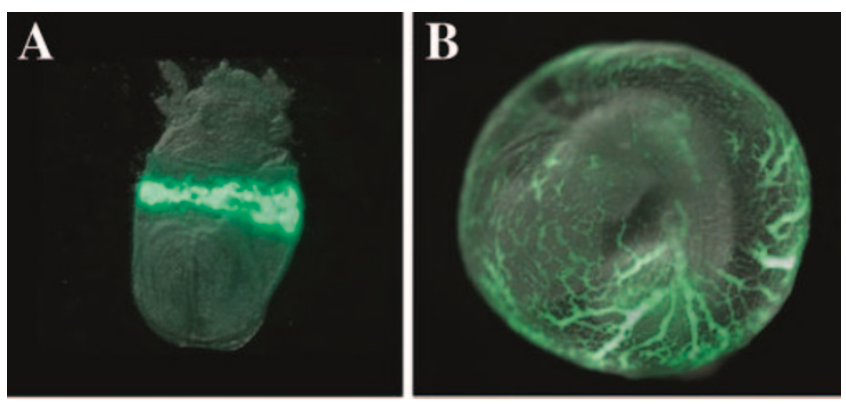

Figure 2. Mouse embryos can be harvested at early $8.5 \mathrm{dpc}$ and placed in culture $(A, 35 \times)$. At this stage, the head-folds are present (bottom of picture) and epsilon-globin:GFP-expressing erythroblasts are confined to the blood islands. After $24 \mathrm{~h}$ in culture, the yolk sac vasculature remodels into elongated conduit arteries and veins that communicate with the embryo proper and distally bifurcate into the small-diameter capillary plexus of the vitelline bed $(B, 20 \times)$. The phenotype of embryos cultured from early somite-stage embryos (approximately $8.5 \mathrm{dpc}$ ) through yolk-sac remodeling (approximately $9.5 \mathrm{dpc}$ ) can be indistinguishable from normally gestating pups of the same age.

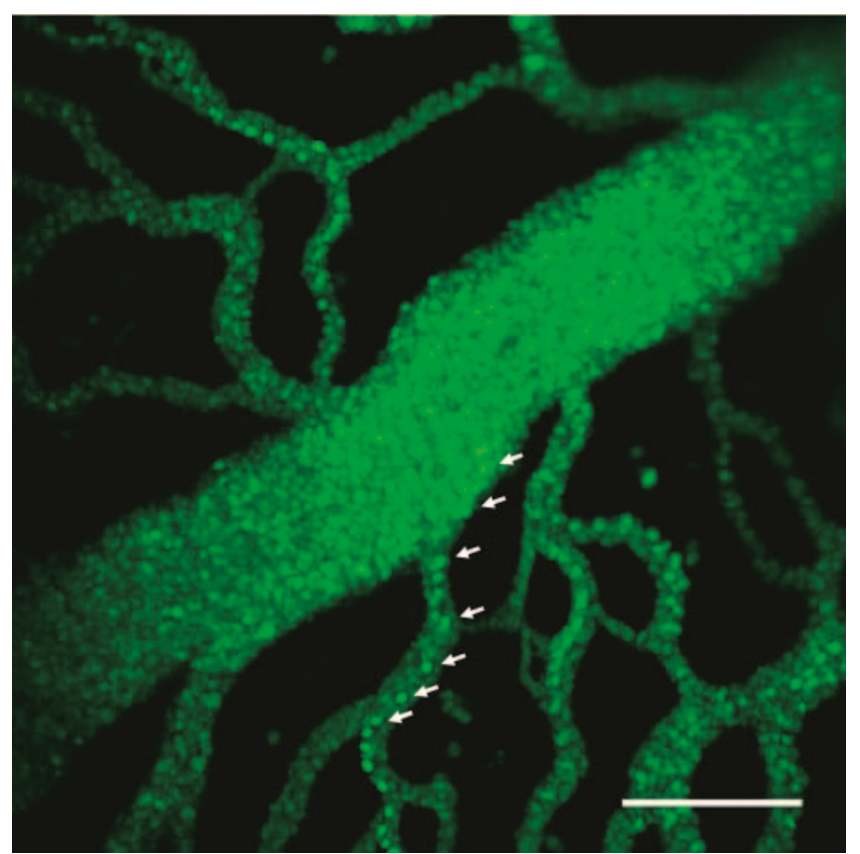

Figure 3. A time-lapse composite image of a vitelline artery of a late $9.5 \mathrm{dpc}$ epsilon-globin:GFP mouse. A vascular cast can be created using slower scanning speeds and is useful for determining vessel diameters and branching morphology. Using higher-speed scanning, individual erythrocytes can be tracked (white arrows) and using very-high-speed scanning, hemodynamic parameters such as flow velocity and shear stress can be calculated. Images were taken every $65 \mathrm{~ms}$ and the bar is $100 \mu \mathrm{m}$.

dynamic parameters in embryos and vessels too small for more "traditional" servo-null or pulsed Doppler techniques.

In the developing nervous system, FP reporters have been instrumental in studying aspects of neuronal differentiation and synapse formation. For instance, "leader" and "follower" axon kinetics and growth cone morphology during zebrafish postoptic commissure establishment is easily followed over time using transgenic gata2:GFP fish (9). Kulesa et al. (10) used four different fluorescent proteins to label migrating avian neural crest cells as they exit the neural tube. This multispectral approach makes it possible to identify and follow individual cells as they come together, move apart, and cross each other's paths. A similar approach with multicolored neurons is used to study the stabilization of synapse formation at the neuromuscular junction. For example, the process of axonal competition for a single synapse in the sternomastoid muscle can be vividly demonstrated by imaging the same region multiple times over the course of weeks in living, intact mouse pups (11).

As our study of development has moved to the molecular level, FP are now being used to investigate molecular interactions between individual proteins. A powerful approach to study whether two proteins interact with each other is through the use of FRET. This is a process in which energy from a fluorophore in an excited state (the donor) is transferred to a fluorophore in a ground state (the acceptor) through a nonradiative exchange, elevating the latter to an excited state. In order for FRET to occur, the donor must emit wavelengths that overlap with the absorption spectrum of the acceptor, the dipole orientations of the two must be reasonably aligned to 
favor energy transfer, and the chromophores must be in very close proximity $(1-10 \mathrm{~nm})$. This latter condition makes FRET an excellent technique to resolve intra- and intermolecular interactions that would otherwise not be possible with standard microscopy techniques. Two of the most popular donor and acceptor molecules are the cyan and yellow FP, including the latest versions, cerulean and venus, respectively. The spectral characteristics of these FP make them ideal for FRET studies and the ease at which one can engineer fusion proteins that can be reintroduced into cells or organisms explain their popularity. Recent applications of these methods include assessing intramolecular distances $(12,13)$, determining conformational changes in activated proteins $(13,14)$, protein-protein interactions (15-17), elucidating phosphorylation-driven signaling pathways $(18,19)$, and measuring intracellular calcium concentrations via the use of genetically modified calmodulin $(20,21)$. FRET has been measured using wide-field, confocal, multispectral, and multiphoton microscopy and, recently, FRET was used to examine the activity of calpain, a cysteine protease, 200 um deep in live mouse tissue (22) using multiphoton microscopy.

\section{BEYOND FLUORESCENT PROTEINS}

Although FP have become a mainstay in the developmental biology community, other probes may offer sophisticated capabilities FP can't yet provide. For instance, MB, first developed in 1996 (23), are probes designed to fluorescently label complimentary DNA or RNA sequences. They consist of a single-stranded oligonucleotide probe flanked on both sides with complimentary sequences that make up the stem. The $3^{\prime}$ end of the strand contains a fluorescent reporter and the $5^{\prime}$ end has a fluorescent quencher. In a nonactivated state, the entire strand has a hairpin-like structure due to the annealing of the complimentary stem sequences. This conformation places the fluorophore and the quencher in close proximity and the fluorophore energy is transferred to the quencher and "silenced." When the beacon encounters a complimentary nucleic acid sequence, the MB unfolds and anneals. This physically separates the fluorophore and the quencher and allows the fluorescent emission to be detected. With a high signalto-noise ratio and a high degree of specificity, MB have been useful for real-time PCR monitoring, DNA analysis, and a number of other applications (24-27).

In living cells, MB have proven useful in tracking mRNA transcription and location $(23,28-30)$. Bratu et al. (31) customized MB to not only track the distribution of oscar mRNA in Drosophila oocytes but to show the translocation of this mRNA from nurse cells to the posterior cortex of the adjacent oocyte to clarify the mechanism of transport of this mRNA. The MB sequence can be flexibly designed to accommodate specific experimental questions and overcome technical challenges presented by living cells. For example, Mlanga et al. (32) observed a tendency for MB to become quickly sequestered by the nucleus and showed that by inserting a tRNA sequence in the MB design, export was facilitated and cytoplasmic MB concentrations were maintained. In combination with FRET technology, MB are a powerful tool that can be used to assess dynamic intracellular interactions in real time.

Another new advancement in fluorescent labeling has emerged as a bioproduct of semiconductor processing. Inorganic nanocrystals, also called QD, are comprised of a crystalline core of semiconductor material, such as CdSE, CdS, or $\mathrm{CdTe}$, coated with a shell that can be functionalized for conjugation with proteins, lipids, or other moieties. Whereas organic fluorophores are limited by low emission intensity, relatively large size, and a propensity for photobleaching, quantum dots emit extremely bright luminescence, are nanometers in diameter, and are extremely photostable. Furthermore, variations in size of the core produce dots that emit at different, very defined wavelengths yet have a wide excitation spectra, making them ideal for multicolor applications (33-36).

Although there have been some initial issues regarding dots clumping and a lack of hydrophilicity, QD continue to rise in popularity. In addition to becoming stable alternatives to traditional fluorochromes for antibody labeling, QD have begun to be used to label living cells and in living embryos. Recently, QD have been conjugated to EGF without disrupting their ability to bind to their receptor, ErbB1, a receptor tyrosine kinase involved with cardiac cushion development. Delivery of the QD-labeled EGF showed EGF situated on cellular filopodia (37). Researchers were then able to investigate the mechanism of EGF/ErbB1 endocytosis and retrograde transport within the cell, and document the transport of individual receptor-ligand complexes along GFP-labeled actin.

\section{CONCLUSION}

A new frontier in developmental biology has emerged following the sequencing of the human genome and the genomes of popular model organisms. Fluorescence imaging remains at the forefront of studies to understand the molecular hierarchies used to control cell identity and behavior during development. Continued effort in the development of more advanced imaging tools, including more functional probes, is needed to add to the toolbox of the developmental biologist. Success in this area will no doubt continue through the synergistic efforts of physicists, biologists, computer scientists, and materials engineers and, while there is a huge effort toward imaging approaches for basic research, translational approaches are on the horizon as more effort is placed on the development of probes for multimodal imaging. The future developmental biology and pediatric diagnosis is bright indeed.

\section{REFERENCES}

1. Centonze VE, White JG 1998 Multiphoton excitation provides optical sections from deeper within scattering specimens than confocal imaging. Biophys J 75:2015-2024

2. Dickinson ME 2005 Multiphoton, multispectral laser scanning microscopy. In: Goldman RD, Spector DL (eds) Live Cell Imaging: A Laboratory Manual. Cold Spring Harbor Laboratory Press, New York

3. Fraser ST, Hadjantonakis AK, Sahr KE, Willey S, Kelly OG, Jones EA, Dickinson ME, Baron MH 2005 Using a histone yellow fluorescent protein fusion for tagging and tracking endothelial cells in ES cells and mice. Genesis 42:162-171

4. Lawson ND, Weinstein BM 2002 In vivo imaging of embryonic vascular development using transgenic zebrafish. Dev Biol 248:307-318

5. Motoike T, Loughna S, Perens E, Roman BL, Liao W, Chau TC, Richardson CD, Kawate T, Kuno J, Weinstein BM, Stainier DY, Sato TN 2000 Universal GFP reporter for the study of vascular development. Genesis 28:75-81 
6. Isogai S, Lawson ND, Torrealday S, Horiguchi M, Weinstein BM 2003 Angiogenic network formation in the developing vertebrate trunk. Development 130:5281-5290

7. Forouhar AS, Liebling M, Wolleschensky R, Ankerhold R, Zimmermann B, Fraser SE, Gharib M Dickinson ME Rapid, confocal microscopy reveals functional changes in embryonic heart development. (in press)

8. Jones EA, Baron MH, Fraser SE, Dickinson ME 2004 Measuring hemodynamic changes during mammalian development. Am J Physiol Heart Circ Physiol 287:H1561-H1569

9. Bak M, Fraser SE 2003 Axon fasciculation and differences in midline kinetics between pioneer and follower axons within commissural fascicles. Development 130:4999-5008

10. Teddy JM, Lansford R, Kulesa PM 2005 Four-color, 4-D time-lapse confocal imaging of chick embryos. Biotechniques 39:703-710

11. Walsh MK, Lichtman JW 2003 In vivo time-lapse imaging of synaptic takeover associated with naturally occurring synapse elimination. Neuron 37:67-73

12. Gordon GW, Berry G, Liang XH, Levine B, Herman B 1998 Quantitative fluorescence resonance energy transfer measurements using fluorescence microscopy. Biophys J 74:2702-2713

13. Chigaev A, Buranda T, Dwyer DC, Prossnitz ER, Sklar LA 2003 FRET detection of cellular \{alpha\}4-integrin conformational activation. Biophys J 85:3951-3962

14. Lee NY, Koland JG 2005 Conformational changes accompany phosphorylation of the epidermal growth factor receptor C-terminal domain. Protein Sci 14:2793-2803

15. Truong K, Ikura M 2001 The use of FRET imaging microscopy to detect proteinprotein interactions and protein conformational changes in vivo. Curr Opin Struct Biol 11:573-578

16. Herrick-Davis K, Grinde E, Harrigan TJ, Mazurkiewicz JE 2005 Inhibition of serotonin 5-HT2C receptor function through heterodimerization: receptor dimers bind two molecules of ligand and one G-protein. J Biol Chem 280:40144-40151

17. Wieland GD, Nehmann N, Muller D, Eibel H, Siebenlist U, Suhnel J, Zipfel PF, Skerka C 2005 Early growth response proteins EGR-4 and EGR-3 interact with immune inflammatory mediators NF-\{kappa\}B p50 and p65. J Cell Sci 118:32033212

18. Ananthanarayanan B, Ni Q, Zhang J 2005 Signal propagation from membrane messengers to nuclear effectors revealed by reporters of phosphoinositide dynamics and Akt activity. Proc Natl Acad Sci U S A 102:15081-15086

19. Green HM, Alberola-Ila J 2005 Development of ERK Activity Sensor, an in vitro, FRET-based sensor of extracellular regulated kinase activity. BMC Chem Biol 5:1

20. Truong K, Sawano A, Mizuno H, Hama H, Tong KI, Mal TK, Miyawaki A, Ikura M 2001 FRET-based in vivo $\mathrm{Ca} 2+$ imaging by a new calmodulin-GFP fusion molecule. Nat Struct Biol 8:1069-1073

21. Takatsuka K, Ishii TM, Ohmori H 2005 A novel Ca2 + indicator protein using FRET and calpain-sensitive linker. Biochem Biophys Res Commun 336:316-323
22. Stockholm D, Bartoli M, Sillon G, Bourg N, Davoust J, Richard I 2005 Imaging calpain protease activity by multiphoton FRET in living mice. J Mol Biol 346:215222

23. Tyagi S, Kramer FR 1996 Molecular beacons: probes that fluoresce upon hybridization. Nat Biotechnol 14:303-308

24. Goel G, Kumar A, Puniya AK, Chen W, Singh K 2005 Molecular beacon: a multitask probe. J Appl Microbiol 99:435-442

25. Fang X, Mi Y, Li JJ, Beck T, Schuster S, Tan W 2002 Molecular beacons: fluorogenic probes for living cell study. Cell Biochem Biophys 37:71-81

26. Tan L, Li Y, Drake TJ, Moroz L, Wang K, Li J, Munteanu A, Chaoyong JY, Martinez K, Tan W 2005 Molecular beacons for bioanalytical applications. Analyst 130:1002-1005

27. Tan W, Wang K, Drake TJ 2004 Molecular beacons. Curr Opin Chem Biol 8:547-553

28. Sokol DL, Zhang X, Lu P, Gewirtz AM 1998 Real time detection of DNA.RNA hybridization in living cells. Proc Natl Acad Sci U S A 95:11538-11543

29. Tyagi S, Bratu DP, Kramer FR 1998 Multicolor molecular beacons for allele discrimination. Nat Biotechnol 16:49-53

30. Marras SA, Gold B, Kramer FR, Smith I, Tyagi S 2004 Real-time measurement of in vitro transcription. Nucleic Acids Res 32:e72

31. Bratu DP, Cha B-J, Mhlanga MM, Kramer FR, Tyagi S 2003 Visualizing the distribution and transport of mRNAs in living cells. Proc Natl Acad Sci U S A 100:13308-13313

32. Mhlanga MM, Vargas DY, Fung CW, Kramer FR, Tyagi S 2005 tRNA-linked molecular beacons for imaging mRNAs in the cytoplasm of living cells. Nucleic Acids Res 33:1902-1912

33. Hanaki K, Momo A, Oku T, Komoto A, Maenosono S, Yamaguchi Y, Yamamoto K 2003 Semiconductor quantum dot/albumin complex is a long-life and highly photostable endosome marker. Biochem Biophys Res Commun 302:496-501

34. Ness JM, Akhtar RS, Latham CB, Roth KA 2003 Combined tyramide signal amplification and quantum dots for sensitive and photostable immunofluorescence detection. J Histochem Cytochem 51:981-987

35. Wang HZ, Wang HY, Liang RQ, Ruan KC 2004 Detection of tumor marker CA125 in ovarian carcinoma using quantum dots. Acta Biochim Biophys Sin (Shanghai) 36:681-686

36. Jaiswal JK, Mattoussi H, Mauro JM, Simon SM 2003 Long-term multiple color imaging of live cells using quantum dot bioconjugates. Nat Biotechnol 21:47-51

37. Lidke DS, Lidke KA, Rieger B, Jovin TM, Arndt-Jovin DJ 2005 Reaching out for signals: filopodia sense EGF and respond by directed retrograde transport of activated receptors. J Cell Biol 170:619-626 